\title{
Static polarizability associated with multipole surface plasmons in metallic surfaces
}

\author{
Nuria Barberán and Martí Pi \\ Departament d'Estructura i Constituents de la Matèria Facultat de Física, Universitat de Barcelona, \\ E-08028 Barcelona, Spain
}

(Received 7 July 1994; revised manuscript received 2 November 1994)

\begin{abstract}
An Euler-Lagrange-type equation is solved to different orders for a plane metallic system perturbed by the external operator $\hat{Q}$ used previously in a sum-rule calculation. This approach managed to reproduce to within a few percent the multipole surface-plasmon energies. The jellium model is used for the positive ionic background and local-density functionals for the Hamiltonian. From the zeroth-order Euler-Lagrange equation a self-consistent ground-state density is obtained and used in the first-order equation to obtain the induced density. From the induced density, several aspects of the system response are considered and special attention is focused on the static polarizability.
\end{abstract}

\section{INTRODUCTION}

Recent inelastic reflection electron scattering experiments on smooth $\mathrm{Na}, \mathrm{K}$, and $\mathrm{Cs}$ surfaces $^{1}$ have confirmed the existence of multipole surface plasmons predicted previously by several theoretical works. ${ }^{2}$ These modes are associated with electronic density fluctuations that are peaked at the surface region and have a decreasing oscillating amplitude towards the interior of the metal.

The aim of the present work is to complete the analysis of some of the topics treated in a previous paper, ${ }^{3}$ which addressed the study of the multipole collective surface modes within a sum-rule (SR) calculation. The SR expressions have often been used ${ }^{4-6}$ to discuss global properties of the random-phase approximation spectral distribution or strength function $S(E)$ in terms of its moments. The strength function is the linear response of the system to an external excitation operator $\hat{Q}$. It has been demonstrated $^{7}$ that the two energies $E_{3}$ and $E_{1}$ defined by $E_{3}(Q)=\sqrt{m_{3} / m_{1}}, E_{1}(Q)=\sqrt{m_{1} / m_{-1}}$, where $m_{k}$ is the $k$ th energy weighted moment of $S(E)$, are upper and lower bounds of its centroid and determine an upper bound of its variance. The more collective the nature of the excited state, the closer the values of the $E_{3}$ and $E_{1}$ energies.

Reference 3 was devoted to the calculation of the $E_{3}$ energies often called "the sudden limit." The perturbation is provided by an external operator $\hat{Q}$, concentrated in the surface region. In the present work, with the same operator a variational calculation is performed and some complementary topics related to multipole plasmons are addressed.

\section{SELF-CONSISTENT GROUND STATE AND INDUCED DENSITY}

If $\varepsilon$ is the energy density of the unperturbed system, $\lambda$ is a small parameter and $n$ is the number of electrons per unit volume, then the solution of the Euler equation,

$$
\frac{\partial \varepsilon(n)}{\partial n}+\lambda Q=\mu
$$

provides the ground-state (g.s.) density of the perturbed system. The Lagrange multiplier $\mu$ (the chemical potential) is used to fix the particle density. Atomic units (a.u.) will be used from now on unless otherwise stated. An extended Thomas-Fermi approach for the kinetic term as well as a Slater plus Wigner expressions for the exchange and correlation contributions are chosen within the local-density function $\varepsilon(n) .{ }^{5}$ If the electronic density is expanded in powers of $\lambda, n(\vec{r})=n_{0}(\vec{r})+\lambda n_{1}(\vec{r})+\cdots$, Eq. (1) can be analyzed for different orders of $\lambda$.

In the limit $z \rightarrow-\infty$, the unperturbed g.s. $n_{0}$ is constant and known for a given metal (let's call it $\tilde{n}_{0}$ ), and the chemical potential measured from the bottom of the Coulomb potential inside the metal is given by (see Table I) $\tilde{\mu}=\mu-\left.U(\vec{r})\right|_{z=-\infty}$, where $U(\vec{r})$ is the total direct Coulomb potential.

Figure 1 shows (full line) the g.s. electronic density for $\mathrm{Na}, \mathrm{K}, \mathrm{Rb}$, and $\mathrm{Cs}$ and Fig. 2 for $\mathrm{Al}$. There is a clear difference between the electronic density for alkali metals and that of $\mathrm{Al}$, since no maximum structure near the jellium edge (at $z=0$ ) appears in the case of $\mathrm{Al}$. This fact meant that the electronic density for $\mathrm{Al}$ could be approximated very well by the following function: ${ }^{8}$

$$
n_{0}(z)=\frac{\tilde{n}_{0}}{e^{z / \delta}+1}
$$

with $\delta=0.65$ a.u. (dashed line), in contrast to the case of alkali metals. For comparison, the analytical approximation given by Eq. (2) is also shown for alkali metals. The $\delta$ parameters for $\mathrm{Na}, \mathrm{K}$, and $\mathrm{Cs}$ are taken from the fit of the polynomial approximation to the experimental data in Ref. 1 for the surface monopole modes and the analytical expression obtained using $Q_{s}=e^{i \vec{q} \cdot \vec{\rho}} e^{q z}(\vec{q}$ is a two-dimensional momentum) and Eq. (2) in a SR calculation in the low $q$ limit, given in Ref. 8 by

$$
w(q)=\frac{w_{p}}{\sqrt{2 m^{*}}}[1-q \delta \ln (2)],
$$

where $m^{*}$ is the electronic effective mass and $w_{p}$ the plasma energy.

In the $\mathrm{Rb}$ case, no experimental data were available and the $\delta$ parameter was adjusted to the numerical 
TABLE I. Physical parameters used in the calculations and work functions for alkali metals and Al. $\tilde{n}_{0}$ is the bulk constant electronic density and $\tilde{\mu}$ is given in the text. $\Phi$ is the work function, $\Phi_{L K}$ was calculated by Lang and Kohn (Ref. 9), and $\Phi_{\exp }$ is the experimental data given in the same reference.

\begin{tabular}{lcccccccc}
\hline \hline & $r_{s}$ & $\begin{array}{c}\tilde{n}_{0} \\
\left(\times 10^{-3}\right)\end{array}$ & $\delta$ & $m^{*}$ & $\tilde{\mu}$ & $\begin{array}{c}\Phi \\
\mathrm{eV}\end{array}$ & $\begin{array}{c}\Phi_{\mathrm{LK}} \\
\mathrm{eV}\end{array}$ & $\begin{array}{c}\Phi_{\text {exp }} \\
\mathrm{eV}\end{array}$ \\
\hline $\mathrm{Al}$ & 2.07 & 26.9 & 0.65 & 1.0 & 2.36 & 3.55 & 3.87 & 4.19 \\
\hline $\mathrm{Na}$ & 3.93 & 3.93 & 1.06 & 1.14 & -2.12 & 2.80 & 3.06 & 2.7 \\
\hline $\mathrm{K}$ & 4.86 & 2.08 & 0.95 & 1.30 & -2.36 & 2.52 & 2.74 & 2.39 \\
\hline $\mathrm{Rb}$ & 5.20 & 1.70 & 0.76 & 1.40 & -2.39 & 2.43 & 2.63 & 2.21 \\
\hline $\mathrm{Cs}$ & 5.62 & 1.34 & 0.60 & 1.58 & -2.39 & 2.33 & 2.49 & 2.14 \\
\hline \hline
\end{tabular}

solution of Eq. (1). All $\delta$ values are summarized in Table I.

Work functions defined as $\Phi=U_{0}-\tilde{\mu}$ [where $U_{0}$ is given by $U_{0}=U(z=\infty)-U(z=-\infty)$ ] are shown in Table I and compared with previous calculations by Lang and $\mathrm{Kohn}^{9}$ and experimental data given in the same reference. The satisfactory matching of our results with experimental data justifies the use of the local density models in the energy functional, except in the case of Al. The difference of our results and those of Ref. 9 is produced by the kinetic term (in Ref. 9 the kinetic contribution is exactly calculated), since the exchange and correlation functionals are the same. That is to say, the nonlocality of the exchange and correlation terms must cancel part of the improvement made in the kinetic term in Ref. 9.

From the first order contribution of Eq. (1), the induced density is obtained. Two different perturbing operators $Q$ are used in order to compare their effects. The operator that generates monopole surface plasmons ${ }^{6,8}$ $Q_{s}=e^{i \vec{q} \cdot \vec{\rho}} e^{q z}$ and the operator that generates multipole $\operatorname{modes}^{3} Q_{m}=e^{i \vec{q} \cdot \vec{\rho}} e^{-q^{2} z^{2}} \cos (\tau z)$.

There are two parameters $(\vec{q}$ and $\tau)$ that characterize the operator $Q_{m}$, one that generates momentum parallel to the surface and which can be varied continuosly and

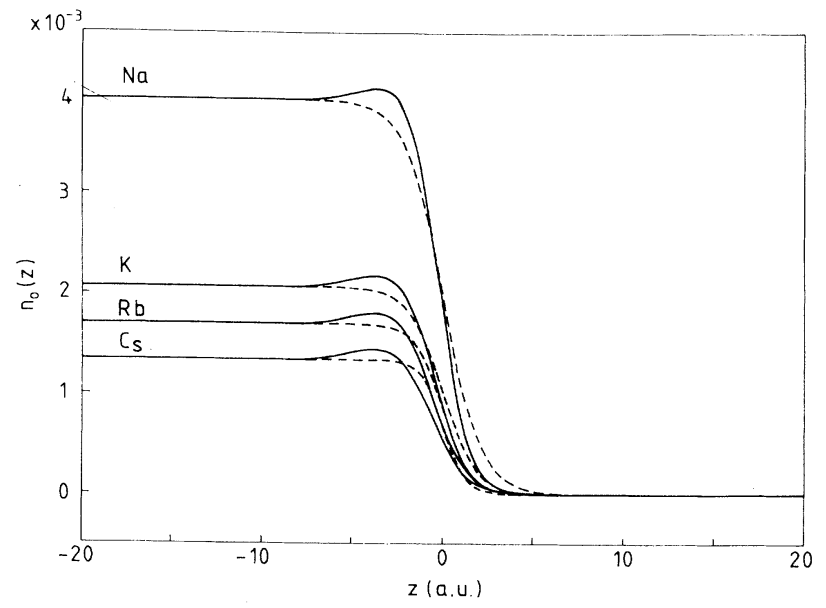

FIG. 1. Self-consistent g.s. densities for alkali metals (full line) and the analytical approach given in Eq. (2) (dashed line). The values of the necessary parameters are summarized in Table I. another related to momentum along the $z$ direction in a standing wave. As seen in Ref. 3, for a fixed value of $q, \tau$ is quantized and only the first quantized value of $\tau$ (let's call it $\tau_{q}$ ) generates observable modes.

Figure 3 shows the multipole mode induced density as a function of $z$ for $\mathrm{Na}$ (dashed line) for $q=0.05$ a.u. and $\tau_{q}=0.086$ a.u. As a comparison, the monopole induced density (for the same $q$ value) is also represented (full line). The integral of $f(z)$ [defined as $n_{1}(\vec{r})=e^{i \vec{q} \cdot \vec{\rho}} f(z)$ ] over the $z$ axis does not vanish, as it should do, because there is a mixture of collective and particle-hole excitations, this mixture is more important for large $q$ values. The $z$ integral diminishes as $q$ decreases and the centroid, defined as

$$
d=\int z f(z) d z
$$

is pushed slightly inwards as $q$ decreases.

Finally, in order to compare the adiabatic induced densities shown in Fig. 3 with the ones obtained from the sudden response of the system, given by ${ }^{5} n_{s}(z)=$ $-\nabla \cdot\left(n_{0} \nabla Q\right)$, we show in Fig. 4 the corresponding result. The multipole character (zero integral along the $z$ direction) is better fulfilled in the sudden case by a factor of $10^{-3}$ and the centroid is shifted towards the interior of the metal.

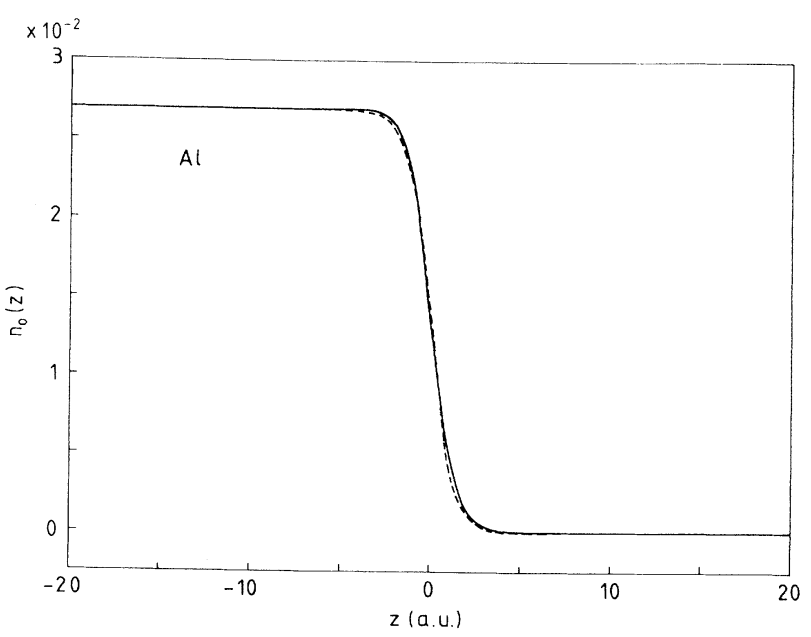

FIG. 2. Same as Fig. 1 for Al. 


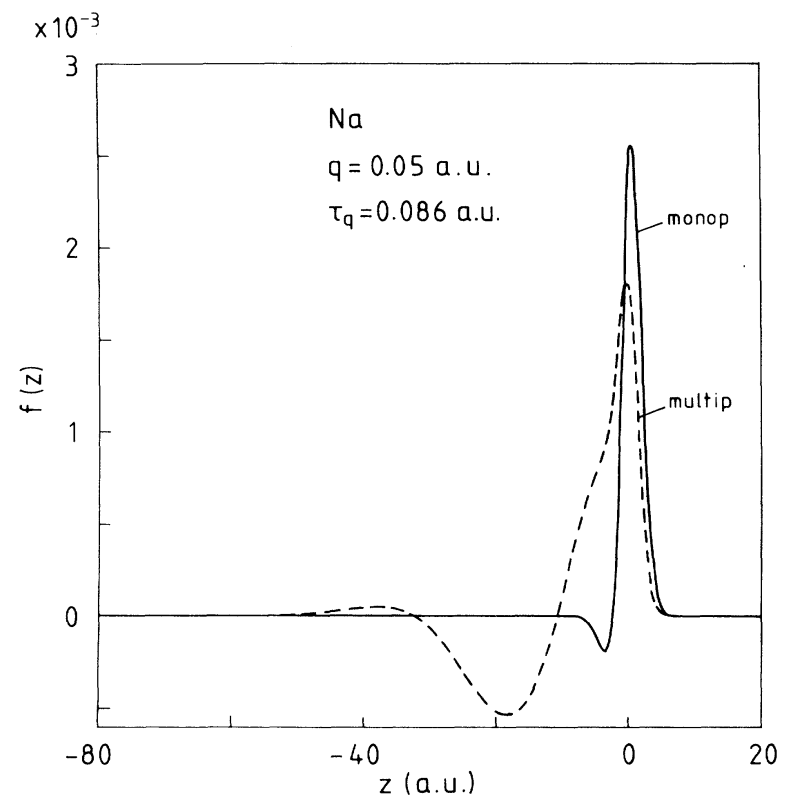

FIG. 3. Function $f(z)$ (see text) for monopole (full line) and multipole (dashed line) modes for Na. $q=0.05$ a.u. and $\tau=0.086$ a.u.

\section{STATIC POLARIZABILITY}

The $m_{-1}$ sum rule is given by ${ }^{10}$

$$
m_{-1}=-\frac{1}{2} \int_{-\infty}^{\infty} d z f(z) g(z)
$$

where $g(z)$ has been defined as $Q=e^{i \vec{q} \cdot \vec{r}} g(\vec{r})$. A rather simple calculation of the $m_{1}$ SR (Ref. 3) allows the calculation of the $E_{1}$ adiabatic energy function. The comparison between $E_{1}$ and $E_{3}$ as functions of $\tau$ and $q$ gives information about the collective nature of the modes.

An effective mass has been incorporated to the $E_{1}$ calculation to include lattice effects. The values of $m^{*}$ (for $\mathrm{Na}, \mathrm{K}$, and $\mathrm{Cs}$ ), summarized in Table I, were taken from

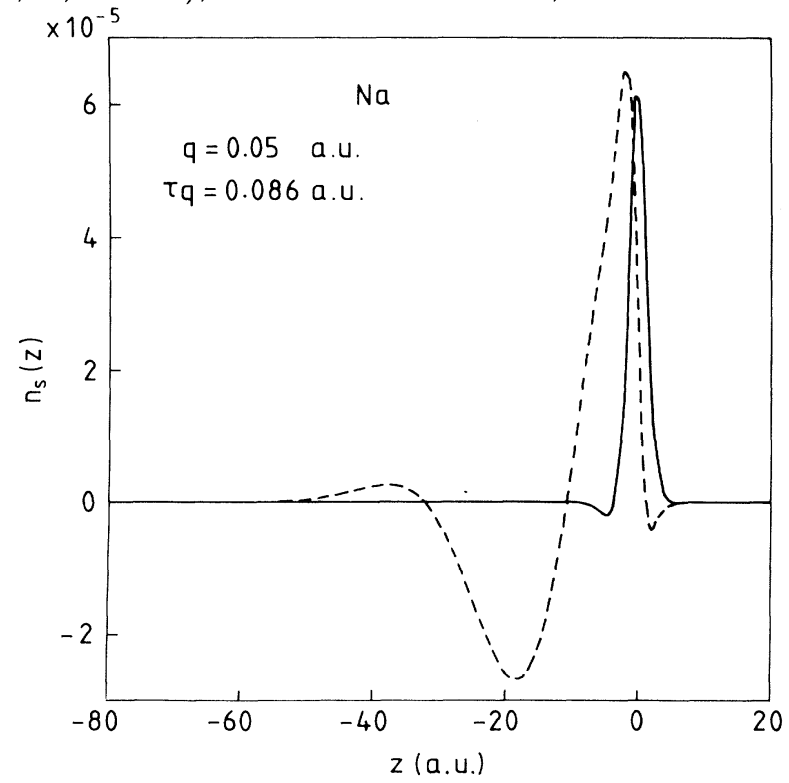

FIG. 4. Induced density in the sudden regime, same notation as in Fig. 3.

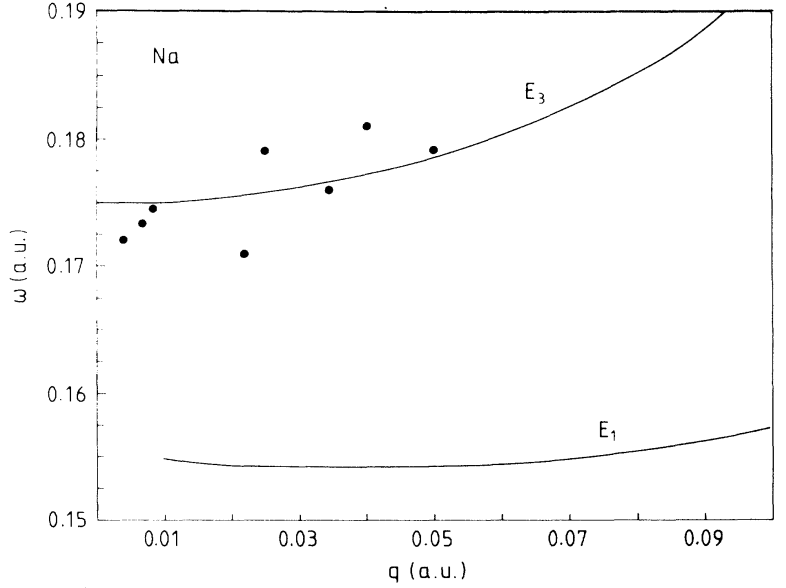

FIG. 5. Sudden and adiabatic energies for $\mathrm{Na}$ as functions of $q$ for the multipole mode. Points represent experimental data from Ref. 1.

the fit of the experimental values of the monopole surfaceplasmon energies at $q=0$ from Ref. 1 and Eq. (3) for $\mathrm{Al}$ and $\mathrm{Rb}$ are trial values. $E_{3}$ is taken directly from Ref. 3 . Figure 5 shows $E_{1}$ and $E_{3}$ as functions of $q$ for $\mathrm{Na}$. The variance of the spectral distribution, determined by the difference between $E_{1}$ and $E_{3}$, increases for larger values of $q$ as collective modes are mixed with electron-hole excitations. Experimental data from Ref. 1 are also shown for comparison.

Figure 6 shows the variation of $E_{1}$ and $E_{3}$ with $\tau$ for $q=0.02$ a.u. The resonant value of $\tau$ is marked below by an arrow. It lies in the region of the lowest difference, between the two calculated energies, and, consequently, of lowest mixture with electron-hole excitations. The collective mode saturates most of the $m_{1}$ SR.

Information about the induced potential can also be obtained from the $f(z)$ function by the equation

$$
\begin{aligned}
\Phi_{\text {ind }}(z, q)= & -\frac{2 \pi}{q} \int d z^{\prime} n_{1}\left(z^{\prime}, q\right) e^{-q\left|z-z^{\prime}\right|} \\
& +\frac{\delta}{\delta n}\left(V_{\mathrm{xc}}[n]\right)_{n_{0}(z)} n_{1}(z, q)
\end{aligned}
$$

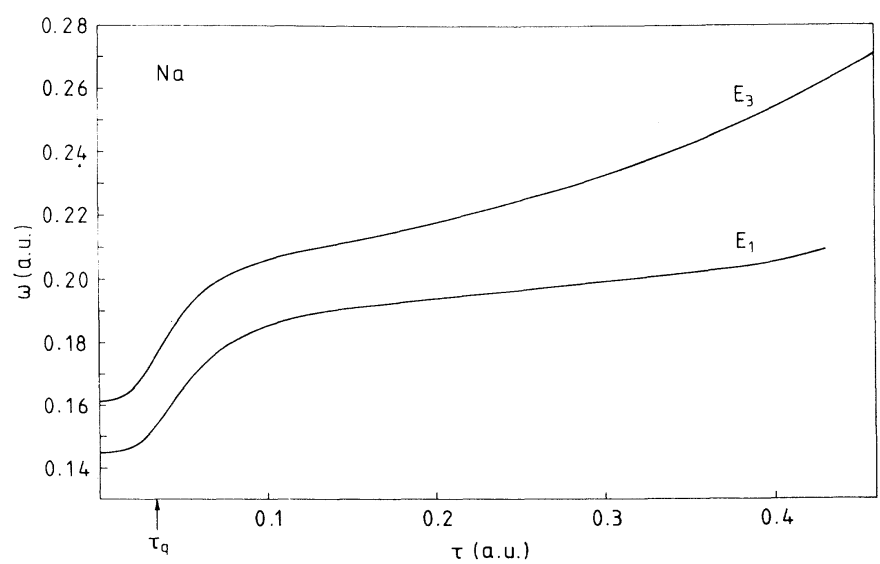

FIG. 6. Sudden and adiabatic energies for $\mathrm{Na}$ as functions of $\tau$ for the multipole mode. The resonant $\tau$ value is marked below by an arrow $q=0.02$ a.u. 
where $V_{\mathrm{xc}}$ is the potential obtained from ${ }^{11} V_{\mathrm{xc}}[n]=$ $\frac{\delta}{\delta n} \varepsilon_{\mathrm{xc}}(n)$, where $\varepsilon_{\mathrm{xc}}$ is the exchange-correlation contribution to the electronic energy density. For monopole modes, $\Phi_{\text {ind }}$ is peaked at a positive $z$ value in such a way that the greater the value of $q$, the lower the overlap between the g.s. electronic density and the induced potential, producing a negative slope in the monopole surface dispersion relation. In the multipole case, it has nodes along the $z$ axis and is peaked slightly inside the metal (as a consequence of this, a positive slope of the dispersed energy results).

Finally, taking advantage of the simple relation between the $m_{-1} \mathrm{SR}$ and the static polarizability, given by $\alpha(q)=-2 m_{-1}(q)$, a study of the variation of $\alpha$ as a function of $q$ and $r_{s}$ has been made. A nearly linear variation of the polarizability (multipole case) with the momentum $q$ is obtained. This behavior agrees with the classical result obtained for monopole modes given by, $\alpha_{\mathrm{Cl}}(q)=-\frac{q}{2 \pi}$. There is even a similarity between the slopes (0.22 and 0.16 a.u. for multipole and monopole, respectively). Furthermore, this classical value of $\alpha$ (and $m_{-1}=\frac{q}{4 \pi}$ ) together with the one obtained for $m_{1}$ in the same classical limit given by ${ }^{3} m_{1}=\frac{q n_{0}}{2}$, produces the nondispersed classical value of the ordinary surface plasmon energy, $E_{1}=\frac{w_{p}}{\sqrt{2}}$. A very different result is obtained for the multipole case if the same procedure is followed to obtain the classical undispersed plasmon energy.

In the multipole case, if the kinetic, exchange, and correlation terms are removed from Eq. (1) the solution for $f(z)$ is no longer a $\delta$-type function as one would expect for a classical incompressible system; that is to say, there is not the classical limit for these multipole modes or in other words, the multipole mode energy at $q=0$ is not of a purely Coulombic nature, as is the case for ordinary surface or bulk plasmons, but instead it has a residual kinetic contribution that makes the $w(q=0)$ value greater than the typical surface frequency $w_{p} / \sqrt{2}$.

Figure 7 compares the variation of the static polarizability (as a function of $r_{s}$ ) of monopole and multipole modes. They behave quite differently; the denser an electron gas, the greater the polarization by monopole perturbations, while in the case of multipole perturbations the opposite is true.

\section{CONCLUDING REMARKS}

The comparison of $E_{1}$ and the sudden regime energy $E_{3}$ calculated in a previous work provides information

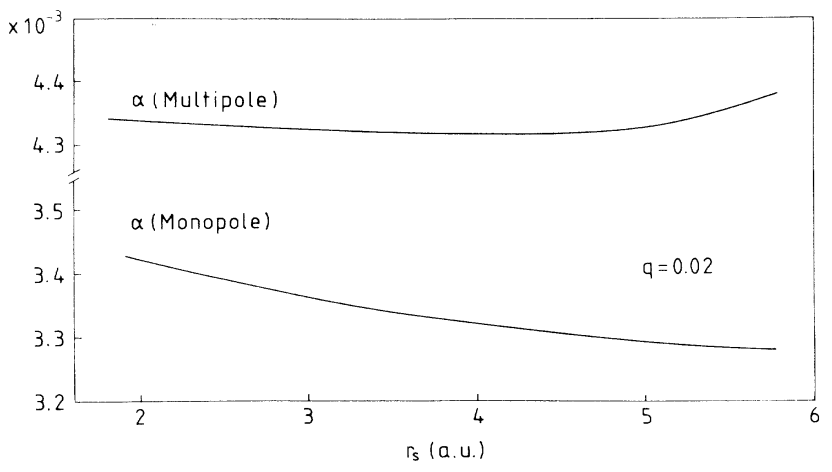

FIG. 7. Comparison of monopole and multipole static polarizabilities as functions of $r_{s}$.

about the variance of the excitation spectrum and also of the adequacy of the operator $Q$ in selecting eigenmodes. As Fig. 5 shows that the $E_{3}$ energy is closer to the experimental data, we infer that the main structure of peaks of the spectral function $S(E)$ is located at high energies.

Although some of the results obtained for $\mathrm{Al}$ are different from those results obtained for alkali metals, as is the case of the g.s. density, the induced density (larger for monopole modes) and the low static polarizability, they do not justify the absence of direct experimental inelastic electron scattering results for multipole modes. Taking into account that a large electronic diffuseness at the surface [given by the $\delta$ parameter in an analytical approximation of the g.s. using Eq. (2)] gave approximate results for the surface monopole modes in Ref. 8 where a trial $\delta=1.60$ a.u. was used, we conclude that the jellium model is not appropriate for representing surface lattice layers in the case of dense free-electron-like metals and that a more realistic inclusion of the ionic background, especially at the surface region is necessary to increase the diffuseness of the electronic density otherwise treated by a steep function of $z$ [see Fig. (2)] in a self-consistent jellium calculation. This increase of the electronic diffuseness would increase the calculated value of the work function improving its fit with experimental results (see Table I).

\section{ACKNOWLEDGMENTS}

We would like to thank Professor J. Martorell, Professor M. Barranco, and Professor R. Tarrach for many helpful discussions. This work has been supported in part by the Comisión Asesora de Investigación Científica y Técnica (CAICyT), Spain under Grant No. PB92-0761.
${ }^{1}$ K.D. Tsuei, E.W. Plummer, A. Liebsch, K. Kempa, and P. Bakshi, Phys. Rev. Lett. 64, 44 (1990).

${ }^{2}$ J. Bennet, Phys. Rev. B 1, 203 (1970); J. Harris and A. Griffin, Can. J. Phys. 48, 2592 (1970); A. Eguiluz and J.J. Quinn, Phys. Lett. 53A, 151 (1973); J.E. Inglesfield and E. Wikborg, J. Phys. C 6 L158 (1973).

${ }^{3}$ J. Sellarés and N. Barberán, Phys. Rev. B 50, 1879 (1994).

${ }^{4}$ G.F. Bertsch and W. Ekardt, Phys. Rev. B 32, 7659 (1985).

${ }^{5}$ Ll. Serra, F. Garcias, M. Barranco, N. Barberán, and J. Navarro, Phys. Rev. B 41, 3434 (1990).
${ }^{6}$ E. Lipparini and F. Pederiva, Z. Phys. D 22, 553 (1992).

${ }^{7}$ O. Bohigas, A.M. Lane, and J. Martorell, Phys. Rep. 51, 267 (1979)

${ }^{8}$ N. Barberán, J. Sellarés, and J. Bausells, Surf. Sci. 292, 159 (1993).

${ }^{9}$ N.D. Lang and W. Kohn, Phys. Rev. B 3, 1215 (1971).

${ }^{10} \mathrm{P}$. Ring and P. Schuck, The Nuclear Many Body Problem (Springer-Verlag, New York, 1980).

${ }^{11}$ A. Liebsch, Phys. Rev. B 32, 6255 (1985). 